\title{
Extended workdays: Effects on performance and ratings of fatigue and alertness
}

\author{
ROGER R. ROSA \\ U.S. Department of Health and Human Services, Centers for Disease Control, National Institute for \\ Occupational Safety and Health, Division of Biomedical and Behavioral Sciences, Cincinnati, Ohio \\ DANIEL D. WHEELER and JOEL S. WARM \\ University of Cincinnati, Cincinnati, Ohio \\ and \\ MICHAEL J. COLLIGAN \\ U.S. Department of Health and Human Services, Centers for Disease Control, National Institute for \\ Occupational Safety and Health, Division of Biomedical and Behavioral Sciences, Cincinnati, Ohio
}

\begin{abstract}
The present investigation provided a laboratory test of long workdays and served as an initial step in developing a field-test battery that is sensitive to fatigue. Six subjects worked both a 12h/4-day workweek and an 8-h/6-day week at a data-entry job simulation. Before and after the first and last days of each week, they completed a battery of brief tests measuring cognitive, perceptual-motor, task-sharing, motor, and sensory capacities, as well as subjective feelings. Results suggest that the 12-h/4-day week was more fatiguing than the 8-h/6-day week. In the data-entry job, it was easier to improve performance across the 8-h/6-day week. This result suggested that the fatigue of the 12-h days slowed the rate of improvement across the week. With respect to the test battery, two cognitive tasks (grammatical reasoning and digit addition) and several selfreport scales also reflected greater fatigue in the 12-h/4-day week. Performance efficiency decreased and reports of drowsiness and lack of concentration increased from the beginning to the end of the final 12-h workday. On the basis of these results, it was concluded that the test battery has utility for the assessment of the potential fatigue effects of long workdays in actual work settings.
\end{abstract}

Employee demands for increased "usable" leisure time and evolving industrial needs for longer work shifts have stimulated interest in alternatives to the common 8-h/5day workweek (e.g., Hedges, 1971). Alternative schedules often include work shifts longer than $8 \mathrm{~h}$ because the 5-day week is "compressed" into 4 days or fewer or because overtime is required. The increased use of longer workdays under such schedules has led to concerns about reduced job efficiency and related problems involving safety and health hazards attributable to cumulative fatigue. The literature on work scheduling (see Alluisi \& Morgan, 1982, for a review) provides only a few clues about the effects of long workdays on performance efficiency or individual well-being.

Studies examining the effects of the compressed workweek have reported inconsistent changes (i.e., increases in some cases and decreases in others) in absenteeism (e.g., Campbell, 1980), worker satisfaction (e.g., Nord

Roger R. Rosa and Michael J. Colligan's mailing address is: U.S. Department of Health and Human Services, Centers for Disease Control, National Institute for Occupational Safety and Health, Division of Biomedical and Behavioral Sciences, Cincinnati, OH 45226. Daniel D. Wheeler and Joel S. Warm's mailing address is: Department of Psychology, University of Cincinnati, Cincinnati, OH 45221.
\& Costigan, 1973), and productivity (Ivancevich, 1974; Ivancevich \& Lyon, 1977). Furthermore, it has been suggested that, in situations in which production levels have been maintained following conversion to a compressed schedule, this may have been at the expense of increased fatigue as reported by workers (Hodge \& Tellier, 1975). In addition, there are few data on how the workers' subjective reports related to actual behavioral measures of work efficiency or safety. There are some reports (e.g., Ellingstad \& Heimstra, 1970; Grandjean, Wotzka, Schaad, \& Gilgen, 1971) of demonstrated declines in performance across a work shift of 8 or more hours, but these studies have not systematically compared different work schedules in terms of days worked or hours worked per day. Other researchers (see Alluisi \& Morgan, 1982, for a review) have compared performance during various work schedules requiring 12 or $16 \mathrm{~h}$ of work per day. However, these studies usually have involved atypical work-rest cycles, such as $4 \mathrm{~h}$ on duty $/ 4 \mathrm{~h}$ off duty, $4 \mathrm{~h} \mathrm{on} / 2 \mathrm{~h}$ off, or $6 \mathrm{~h}$ on $/ 2 \mathrm{~h}$ off, which were designed to simulate military oprations. The alteration of the normal sleep routine during such short work-rest schedules makes it difficult to apply the results of these studies to the long work-rest cycles, such as $10 \mathrm{~h}$ on/14 h off, common in come civilian occupations. 
Only two research groups (Colquhoun, Blake, \& Edwards, 1968, 1969; Volle, Brisson, Perusse, Tanaka, \& Doyon, 1979) have examined extended work periods similar to those being implemented in civilian settings. Colquhoun and colleagues studied circadian changes in performance during 8- and 12-h shifts under simulated naval operations. Direct comparisons of shift length were not intended in these studies, but there were some suggestions of greater cumulative fatigue under the 12-h shift. Subjects recovered from a transient postlunch decrease in performance more quickly in the 8- than in the 12-h shift. In addition, there was a $49 \%$ improvement in performance across days in the 8-h shift and only a $30 \% \mathrm{im}$ provement across days in the $12-\mathrm{h}$ shift. This difference in rate of improvement occurred in spite of the 12-h group's having $50 \%$ more practice with the task. These results are not conclusive, however, because they were confounded by the inadvertent assignment of different personality types, with known circadian differences in performance, to the different shifts (Colquhoun, personal communication, October 1982).

In the other examination of extended work hours, Volle et al. (1979) used several brief measures to compare manufacturing plant emloyees on a 10-h/4-day week with those on an 8-h/5-day week. At the end of the week, the 10-h/4-day group was significantly less efficient than the 8-h/5-day group on measures of right-hand grip strength and critical flicker fusion theshold.

In summary, there are few direct evaluations of the effects of long workdays on individual functioning. Those that do exist (Colquhoun et al., 1968, 1969; Volle et al., 1979) have provided some suggestion of accumulated fatigue across a number of long workdays. These studies pointed to a need for systematic assessment of long work shifts, beginning with controlled laboratory study and subsequently extending to long-term field study. Information gained from these investigations can aid policy decisions about the implementation of long work shifts.

The first step in this process is the development of a sound methodology for both laboratory and field study. A test battery approach, similar to, but more elaborate than, that used by Volle et al. (1979), seemed to be a viable method. The application of a set of brief standardized measures to evaluate work schedules in various occupations would allow generalization across occupations or work settings. Accordingly, the present investigation was designed to develop and evaluate a methodology for the assessment of performance and subjective fatigue associated with long workdays. We reasoned that, for a fatigue test battery to be effectively used in field research, it should be portable, easy to administer, and brief, so that it could be completed within $1 \mathrm{~h}$. In addition, tasks should be chosen such that they require minimal training on the part of the subject. These criteria were desirable because they minimized interference with the workers' regular jobs. Previous test batteries with demonstrated sensitivity to long hours of sustained operations (Alluisi \& Morgan, 1982; Chiles, Alluisi, \& Adams, 1968) have not met these criteria because they were not portable and they required extensive training and experimenter intervention. All of these aspects make the methods cumbersome to use in the field.

In the present investigation, a battery of performance measures was tested in the laboratory, with subsequent field application as the eventual goal. Tests for a wide range of psychological functions were included in the hope that some could be used as general indices of efficiency and that others could be included or excluded from future field studies on the basis of their compatibility with real-life work tasks (see Monk \& Embrey, 1981, and Volle et al., 1979). The sensitivity of the battery of performance measures was tested by comparison with a quantifiable job simulation under two work schedules requiring time beyond the common $40-\mathrm{h} / 5$-day week. The performance measures were also compared with concurrent indices of subjective fatigue and alertness.

\section{METHOD}

\section{Subjects}

The number of subjects who could participate in the investigation was limited by time and resource considerations. Thus, the use of strict selection criteria was necessary to minimize error variance. Six males (ages 22-27 years) were recruited from a temporary clerical service. All could type at least 40 words per minute, but only three had had experience with the 10-key numerical pad used in the data-entry task. They were selected to be in good physical and mental health, drug-free, infrequent alcohol and tobacco users, and good 7-8-h sleepers for at least 6 months prior to the study. During the study, all subjects were drug-free and restricted alcohol intake to less than $1 \mathrm{oz}$ per day. Subjects were paid $\$ 5.00 / \mathrm{h}(\$ 7.50 / \mathrm{h}$ for overtime) for their services.

\section{Measures of Performance and Subjective State}

Continuous performance was measured with a simulated "real-life" task, and intermittent performance was measured with a battery of brief standardized laboratory tasks designed to evaluate a range of psychological functions, including cognitive abilities, perceptual-motor functions, motor skills, and sensory acuity. Included with the intermittent performance tasks were brief scales of subjective alertness and fatigue.

Both continuous performance and the intermittent performance/self-report battery were controlled by a Kaypro II microcomputer. The Kaypro II was selected from among several microcomputers for its compactness, durability, and portability-features ideally suited for future field studies. Interfaces with the Kaypro II were two small response boxes and a Hayes Stack chronograph (Hayes Microcomputer Products, Inc.). The software for the test battery was designed to be highly flexible for a variety of research applications or settings. This was ac- 
complished in two ways. First, the number, type, sequencing, and timing of existing tasks and scales can be specified for a given test session with a separate control file that is read by a master control program. The control file is easily written with a word processor. Second, new tasks and scales can be added in the future in separate program modules. These modules can be read by the master control program without altering existing software.

\section{Continuous Performance: Data Entry}

The data-entry task was a partial simulation of the type of data-entry work performed by thousands of U.S. clerical workers. Five-digit numbers were constructed randomly and presented on the computer viewing screen to be entered on the keyboard by the subject as quickly as possible. There was no provision for correcting an erroneous code. The subject merely completed that number and proceeded to the next. The task was subject-paced in that no new code was presented until the previous code had been entered. There was, however, a minimum criterion of 160 correct codes per 10 -min period. If performance fell below this criterion, a warning message was presented on the viewing screen following that 10 -min period. The message stated the total number of codes completed, the number of errors, and the final score (trials minus errors). An error was any code with at least one miscopied digit. The task was scored for the number of correctly completed codes and the number of errors per 10-min period. Performance was continuous for periods of 60 or $110 \mathrm{~min}$; breaks were allotted between periods. Length of the periods and breaks depended upon the scheduling of other measurements (see Figure 1).

\section{Intermittent Performance/Self-Report Battery}

The tasks and questionnaires were obtained from a variety of sources, including investigations concerned with work scheduling, biological rhythms, sleep deprivation, and sleep scheduling. Although not all of the measures

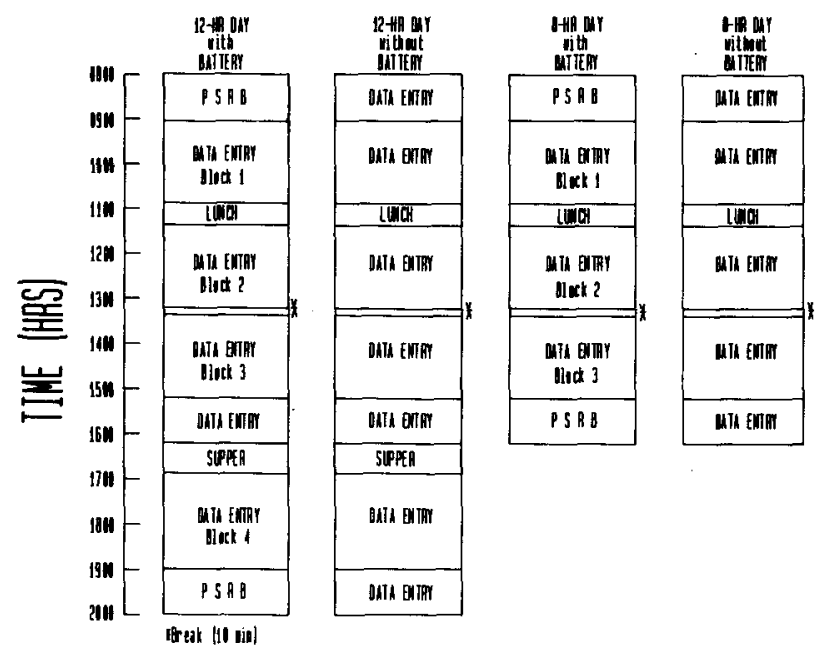

Figure 1. Daily task schedule for 12-h and 8-h days with and without performance/self-report battery (PSRB). have been used in studies focusing specifically on long workdays, the measures share the common theme of being sensitive to changes in CNS activation. This theme, coupled with the feasibility of programming on the microcomputer, guided the selection of performance tasks.

\section{Cognitive abilities.}

Grammatical Reasoning. This 3-min test of verbal analytical abilities is coupled with a short-term-memory requirement and is a variation of a task devised by Baddeley (1968). A two- or three-letter stimulus string (e.g., $\mathrm{JLN}$ ) was initially presented for $2 \mathrm{sec}$, was then removed, and was followed after $2 \mathrm{sec}$ with a conditional satement such as:

\section{J DOES NOT PRECEDE N}

The subject then pressed a push-button switch labeled TRUE or FALSE as quickly as possible to indicate whether the statement satisfied the letter string. To minimize acoustic confusability, only the letters $\mathrm{J}, \mathrm{L}$, and $\mathbf{N}$ were used. Both the letter strings and the conditional statements were generated randomly for each trial. The entire 3-min task was conducted with a memory load of two letters and a memory load of three letters at each testing time. The order of the tasks with each memory load was randomized across subjects. Scores for the task were the mean and standard deviation of the response latencies and the total number of errors for the $3 \mathrm{~min}$.

Digit Addition. This 3-min task was adapted from Williams and Lubin (1966). The subject was required to add two digits presented on the screen and to type the last digit of the sum on the keyboard. In a separate 3-min segment, the subject was required to add a constant to the sum of the two digits and to type the last digit of the final sum on the keyboard. The order of presentation of the tasks (with or without a constant) was randomized across subjects. The subject was asked to work as quickly as possible. The score was the total number of additions completed and the number of errors per 3-min period. The two versions of the task were used so that differences based on the number of cognitive operations (two additions vs. one) could be compared while keeping the stimulus input (two digits) and the response output (a single keystroke) the same.

Williams Word Memory. Two 15-word lists were presented at each testing session for immediate free recall. Stimuli were one- or two-syllable nouns with a frequency of at least 15 per million according to Thorndike and Lorge (1944). Each word was presented on the screen for $5 \mathrm{sec}$. To assure registration, the subject said each word to himself, spelled it, and said it again as it was presented on the viewing screen. The subject then typed as many words as he could recall within 2 min after completion of the list. The score for the task was the total number of words recalled from both lists. The task was adapted from Williams, Geiseking, and Lubin (1966).

Time Estimation. Subjects were asked to produce two 15 -sec intervals and two $30-\mathrm{sec}$ intervals by using a 
keypress as a start and a stop signal. They were requested not to count during the interval. Each time estimate was converted to a ratio of the estimated time divided by the target interval. The score for a given session was the mean of these ratios.

\section{Perceptual-motor functions.}

Simple Auditory Reaction Time. The subject was required to respond as quickly as possible to each of 150 43-msec bursts of white noise. These stimuli were generated by a National Semiconductor M5807 white-noise chip and were presented via headphones at $72 \mathrm{~dB}$ (SPL) for $10 \mathrm{~min}$. The mean interstimulus interval was 4-sec, with a variation of $\pm 2 \mathrm{sec}$. During the task, the subject maintained an upright sitting position and rested the forefinger of his nonpreferred hand on a push-button switch mounted in a response box on a table. Scores for the task were the mean and standard deviation for the entire $10 \mathrm{~min}$ and the number of response times longer than $1.5 \mathrm{sec}$ (lapses or blocks; Bills, 1931).

Choice Reaction Time. Stimuli were 100 random selections of the words TRUE and FALSE presented on the viewing screen for $10 \mathrm{~min}$. The subject responded on similarly labeled push-button switches with the middle and forefinger of his preferred hand. Other aspects of the task were the same as simple auditory reaction time. Scores for the task were the mean and standard deviation for the $10 \mathrm{~min}$, the number of choice errors, and the number of lapses or blocks.

Dual-Task Performance: Grammatical Reasoning and Simple Reaction Time. Given the brief duration and infrequency of the intermittent performance tasks, it was speculated that the subjects might be able to rally enough effort to perform the short tasks at a consistently proficient level. For this reason, the two-letter version of the grammatical reasoning task and the simple auditory reaction time task were also performed simultaneously in an attempt to measure the allocation of spare capacity (see Kahneman, 1973). Bursts of white noise were presented on $60 \%$ of the grammatical reasoning trials, in one of six randomly selected positions in time, prior to the presentation of the conditional statement. In order to minimize response interference, no auditory probes were presented after the conditional statement. Thus, it was assumed that any potential interference attributable to dual-task performance occurred during the processing of the letter string in working memory. Responses to the reasoning task were always made with the preferred hand, and responses to the reaction time task were always made with the nonpreferred hand. The subject was instructed that his primary task was to maintain his best performance on the reasoning task while performing the reaction time task to the best of his ability. All other aspects of the tasks were the same as those tasks performed individually. Allocation of effort was assumed to be reflected in the difference between the scores of the tasks performed simultaneously and the scores of the tasks performed individually.

Sensory acuity: Two-Point Auditory Discrimination. This task is a measure of the temporal resolving power of the auditory system. We developed it as an auditory counterpart to critical flicker fusion. Several pairs of 108msec white-noise pulses, with varying periods of silence between them, were presented via headphones at $72 \mathrm{~dB}$ (SPL). The subject then decided whether the stimulus pairs sounded like one continuous burst of noise or like two bursts of noise, and indicated his judgment by pressing the numeral 1 or 2 on the keyboard. Fusion threshold was determined by the dual-staircase method of limits (Dember \& Warm, 1979). The threshold score was the length (in milliseconds) of the silent gap between the two noise pulses. Discrete pairs of noise pulses were used instead of a continuous on-off series of noise pulses (the analog of a flickering light) to avoid the confusion created by the perception of pitch at the modulating (on-off) frequency of a series of noise pulses:

\section{Motor skills.}

Response Alternation Performance (Tapping). The task required 3 min of repeated, alternating taps on two keys with the middle and forefinger of the preferred hand. It was scored for the total number of taps and for the number of taps with an intertapping interval greater than $750 \mathrm{msec}$ (lapses). The lapse value was based on pilot work with unfatigued subjects and represents the slowest tenth percentile of the total number of taps. The response alternation task was adapted from Naitoh (1981).

Hand Steadiness. The task required centering a conductive metal wand inside a circular metal opening ( $1 / 8$ thin. diameter) for a period of $3 \mathrm{~min}$. During the task, the subject rested his elbow on the arm of the chair. No other support for the hand was allowed. Touching the perimeter of the opening completed a circuit and activated a counter that sampled at a rate of 150 times $/ \mathrm{sec}$. Score for the task was the percentage of time off-target.

Self-report measures. A single program module controlled the presentation and scoring of the scales described below. The questionnaire module handles a number of scaling formats and scoring methods.

Questionnaire for Subjective Symptoms of Fatigue. The fatigue rating scale developed by Yoshitake $(1971,1978)$ has 30 descriptive phrases covering three broad categories of fatigue: drowsiness or dullness, difficulty in concentrating, and somatic complaints. The subject answered yes or no to each phrase, and the total number of yes responses was scored for each category.

Stanford Sleepiness Scale. The 7-point Likert-type scale devised by Hoddes, Zarcone, Smythe, Phillips, and Dement (1973) has descriptors ranging from "very alert" to "very sleepy." The subject was instructed to choose the set of descriptors that best described his feeling of sleepiness at the given moment.

Activation-Deactivation Adjective Checklist. Twentyone adjectives describing activation were presented in a randomized sequence and scored on a 5-point intensity scale ranging from 1 ("not at all") to 5 ("definitely"). The adjectives were originally factor-analyzed into four dimensions: general activation, high activation, general deactivation, and deactivation-sleep (Thayer, 1967). Later 
factor analysis revealed that the general activation and deactivation-sleep categories explained most of the variance (Clements, Hafer, \& Vermillion, 1976).

\section{Design}

Two 48-h work schedules were compared in a repeatedmeasures design. A workweek of 68 -h days and a workweek of 4 12-h days were counterbalanced across subjects, with a 2-day break between each extended workweek. Subjects worked at the continuous data-entry task throughout every day in each workweek. The intermittent performance/self-report battery was given at the beginning and end of Days 1 and 4 of both work schedules. Two nonwork baseline days were given both before and after the experimental regimen to measure circadian rhythm changes in the performance/self-report battery. The postexperiment baseline days occurred at least 3 days after the workweek to minimize carryover effects. One of each of the pre- and postexperiment baseline days consisted of morning $(0800 \mathrm{~h})$ testing, and the other pre- and postexperiment baseline day consisted of evening (1600 and $1900 \mathrm{~h}$ ) testing. Times of testing on baseline days were also counterbalanced across subjects.

\section{Procedure}

The six subjects were seated three to a room in a manner that prevented their viewing one another's performance. Each subject was assigned a Kaypro II microcomputer, which presented all tasks and questionnaires and stored all data. Talking was kept to a minimum during the workday and was prohibited during completion of the performance/self-report battery.

Before the preexperiment baseline sessions, all subjects reported to the laboratory for a session of massed practice to become familiar with all tasks and scales. The dataentry task was performed for $1 \mathrm{~h}$ and the entire performance/self-report battery was performed twice. Order of tasks in the performance/self-report battery was unique for each subject, and that order was maintained for the subject throughout the study.

Morning baseline sessions occurred between 0800 and $0900 \mathrm{~h}$, and evening baseline sessions occurred between 1600 and $1700 \mathrm{~h}$ and between 1900 and $2000 \mathrm{~h}$. The subjects reported to the laboratory at these times and completed the performance/self-report battery. They were then released from the laboratory. During nonlaboratory times on both baseline days and workdays, the subjects engaged only in relaxing activities and avoided napping and overexertion. They also maintained regular sleep times, eating habits, and caffeine use throughout the study.

Schedules for the 8-h and 12-h workdays with the intermittent battery and those workdays without the intermittent battery are presented in Figure 1. Excluding lunch, dinner, and breaks, actual work time during the 8-h days amounted to $7 \mathrm{~h} 30 \mathrm{~min}$, and actual work time during the 12-h days amounted to $10 \mathrm{~h} 20 \mathrm{~min}$. Actual work time included both continuous performance and the intermittent battery.

\section{Results}

An alpha level of .10 was adopted for rejection of the null hypothesis in all statistical analyses. This liberal criterion was chosen because of the small number of subjects and because the test battery is in the early stages of development. Since we were looking for promising leads to sensitive tests, the avoidance of Type II errors was considered more important than the commission of Type I errors.

\section{Continuous Performance: Data Entry}

The number of correctly copied codes per 10-min period (speed of performance) and the number of copy errors per 10-min period (accuracy of performance) were first averaged for each subject within each 110-min time block. This procedure resulted in scores for three time blocks across an 8-h day and four time blocks across a 12-h day (see Figure 1). Both speed and accuracy scores were subjected to analyses of variance comparing performance across workdays between the 8-h/6-day week and 12-h/4day week and simple main effects within each workweek.

Correctly copied codes. Table 1 presents the mean number of correctly copied codes per 10-min period for each group (or order) during the first and last days of the 8-h/6-day week and the 12-h/4-day week. The three subjects in Group 1 experienced the 8-h/6-day week first and then the 12-h/4-day week. The order was reversed for the three subjects in Group 2. As shown in Table 1, performance improved across both weeks. It seemed easier, however, to improve speed across the 8-h/6-day week. Group 1 showed the greatest improvement of either group or workweek in their first week of 8-h days. Their performance then remained stable through their second week of 12-h days. Group 2, however, continued to improve from their first week of 12-h days to their second week of 8-h days. The largest improvement in speed of performance in Group 1 is reflected in a statistically significant interaction of group $\times$ week $\times$ day $[\mathrm{F}(1,4)=25.52$, $p<.01]$. The continued improvement from the first to the second week in Group 2 is reflected in a significant interaction of group $\times$ week $[\mathrm{F}(1,4)=8.48, \mathrm{p}<.05]$. Both the performance of Group 1 and the performance of Group 2 imply that it is easier to increase task effi-

Table 1

Means and Standard Deviations (SDs) for the Number of Correct Codes per 10-min Period

\begin{tabular}{|c|c|c|c|c|c|c|c|}
\hline \multirow[b]{2}{*}{ Group } & \multirow[b]{2}{*}{ Week } & \multicolumn{2}{|c|}{ First Day } & \multicolumn{2}{|c|}{ Last Day } & \multicolumn{2}{|c|}{ Overall } \\
\hline & & Mean & $S D$ & Mean & SD & Mean & SD \\
\hline 1 & 8-h & 126 & $\begin{array}{l}20 \\
15\end{array}$ & 155 & 9 & 143 & 17 \\
\hline 2 & $\begin{array}{r}12-h \\
8-h\end{array}$ & $\begin{array}{l}176 \\
192\end{array}$ & $\begin{array}{l}14 \\
23\end{array}$ & $\begin{array}{l}186 \\
202\end{array}$ & $\begin{array}{l}17 \\
31\end{array}$ & $\begin{array}{l}181 \\
197\end{array}$ & $\begin{array}{l}16 \\
24\end{array}$ \\
\hline
\end{tabular}


Table 2

Number of Correct Codes and Number of Errors per 10-min Period Across the 12-h Day

\begin{tabular}{llrrrr}
\hline & \multicolumn{5}{c}{ Time Block } \\
\cline { 2 - 6 } & & \multicolumn{5}{c}{ 1 } & \multicolumn{1}{c}{ 2 } & \multicolumn{1}{c}{3} & 4 \\
\hline \multirow{2}{*}{ Correct Codes } & Mean & 168 & 164 & 159 & 165 \\
& SD & 20 & 21 & 27 & 23 \\
\multirow{3}{*}{ Errors } & Mean & 13 & 15 & 16 & 15 \\
& SD & 6 & 9 & 8 & 7 \\
\hline
\end{tabular}

ciency in the 8-h week. These results suggest that the 12$\mathrm{h} / 4$-day week was more fatiguing.

In addition to the above results, there was a significant time-of-day effect across the $12-\mathrm{h}$ day $[\mathrm{F}(3,12)=3.15$, $\mathrm{p}<.10]$. Means and standard deviations for each time block across the 12-h day are presented in Table 2 . As shown in Table 2, speed of copying decreased from the first through the third time block and then increased in the last time block of the 12-h day.

Errors. Analyses of variance for number of errors revealed significant effects for week $[F(1,4)=11.96, p<$ $.05]$ and time of day $[F(1,4)=6.18, p<.10]$ in the 12 $\mathrm{h} / 4$-day week. The effect of week reflects the greater number of errors committed in the 12-h/4-day week regardless of group (12 in the 8-h/6-day week vs. 15 in 12-h/4day week). Means and standard deviations for the number of errors across time of day are presented in Table 2 . Errors increased from the first to the third time block and then decreased in the last time block. These results parallel the time-of-day effect for total number of correct codes (see Table 2).

\section{Intermittent Performance/Self-Report Battery}

The design of the present study called for all of the subjects to begin work at the same time of day and, thus, to complete the 8-h and 12-h workdays at times of day separated by $4 \mathrm{~h}$. Since many of the tasks and scales used in the present study are potentially sensitive to circadian variation, it would be possible to obtain differences between the 8- and 12-h days simply on the basis of the different finishing times. It would be difficult, then, to attribute any differences between the 8- and 12-h days to the length of the work period per se. In order to partial out some of the circadian variation in the tasks and scales, each dependent variable was first expressed as a percentage of the nonwork, time-anchored baseline or, when it was possible for zero to be a score (e.g., number of er- rors), as a difference from baseline. This was accomplished by averaging each subject's pre- and postbaseline scores at each time of day to first obtain a mean timeanchored baseline for each subject. The mean baseline controlled for practice effects attributable to repeated exposure to the tasks across the duration of the experiment (see Williams et al., 1966). The workday scores for each subject were then divided by, or subtracted from, the baseline scores at the appropriate time of day. (Note that a positive difference score signifies a value above baseline and a negative difference score signifies a value below baseline.) Each dependent variable in the battery was subject to analyses of variance comparing groups, workweeks, days within weeks, the beginning versus the end of the workshift, and their interactions. Tests of simple main effects within the 8-h/6-day week or within the 12h/4-day week were also conducted. Given the large number of dependent measures, only those variables showing significant differences between the workweeks are presented.

Grammatical Reasoning (three-letter string). Table 3 shows mean response time (expressed as a percentage of baseline) at the beginning and the end of the first and last days of the 8-h/6-day week and the 12-h weeks. It can be seen in Table 3 that response time either decreased or remained stable from the beginning to the end of the 8-h days and increased from the beginning to the end of the 12-h days. These results suggest an increase in fatigue across the 12-h days. The results are supported by a significant interaction of day $\times$ time of $\operatorname{shift}[F(5,20)=4.24$, $\mathrm{p}<.01]$.

Analyses of variance for the within-session standard deviation of response time revealed a significant interaction of group $\times$ week $[F(1,4)=7.49, p<.05]$. This interaction reflects poorer performance (larger standard deviation) in the 12-h/4-day week regardless of group. The magnitude of difference, however, was larger for Group 2 (93\% vs. $76 \%$ for Group 1 and $163 \%$ vs. $109 \%$ for Group 2).

Digit Addition. Table 4 presents means and standard deviations for the total number of correct additions in the version of the task in which no constant was added to the initial sum. These values are shown at each testing time across each day in the 8-h/6-day week and the 12-h/4day week. Scores for the number of correct additions improved from the beginning to the end of the day and from the beginning to the end of the week in the 8-h/6-day week. They declined from the beginning to the end of the

Table 3

Means and Standard Deviations (SDs), Collapsed Over Groups, for Response Time in the Three-Letter Grammatical Reasoning Task (Percent Baseline

\begin{tabular}{|c|c|c|c|c|c|c|c|c|c|c|c|c|}
\hline \multirow[b]{3}{*}{ Time of Day } & \multicolumn{6}{|c|}{ 8-h Week } & \multicolumn{6}{|c|}{ 12-h Week } \\
\hline & \multicolumn{2}{|c|}{ Day 1} & \multicolumn{2}{|c|}{ Day 6} & \multicolumn{2}{|c|}{ Overall } & \multicolumn{2}{|c|}{ Day 1} & \multicolumn{2}{|c|}{ Day 4} & \multicolumn{2}{|c|}{ Overall } \\
\hline & Mean & SD & Mean & SD & Mean & SD & Mean & $\mathrm{SD}$ & Mean & SD & Mean & SD \\
\hline Beginning & 100 & 15 & $\overline{105}$ & 18 & 99 & 19 & 103 & $\overline{21}$ & 104 & 22 & 104 & 21 \\
\hline End & 96 & 18 & 80 & 17 & 89 & 17 & 108 & 16 & 114 & 24 & 111 & 20 \\
\hline Overall Mean & 98 & 16 & 93 & 21 & 94 & 19 & 106 & 18 & 109 & 23 & 108 & 20 \\
\hline
\end{tabular}


Table 4

Means and Standard Deviations (SDs), Collapsed Over Groups, for the Total Number Correct in the Digit Addition Task (No Constant) (Percent Baseline)

\begin{tabular}{|c|c|c|c|c|c|c|c|c|c|c|c|c|}
\hline \multirow[b]{3}{*}{ Time of Shift } & \multicolumn{6}{|c|}{ 8-h Week } & \multicolumn{6}{|c|}{ 12-h Week } \\
\hline & \multicolumn{2}{|c|}{ Day 1} & \multicolumn{2}{|c|}{ Day 6} & \multicolumn{2}{|c|}{ Overall } & \multicolumn{2}{|c|}{ Day 1} & \multicolumn{2}{|c|}{ Day 4} & \multicolumn{2}{|c|}{ Overall } \\
\hline & Mean & $\mathrm{SD}$ & Mean & SD & Mean & SD & Mean & SD & Mean & SD & Mean & SD \\
\hline Beginning & 97.6 & 6.8 & 99.4 & 3.5 & 98.8 & 4.6 & 100.3 & 1.4 & 100.0 & 3.5 & 100.2 & 2.6 \\
\hline End & 97.1 & 6.4 & 100.7 & 2.6 & 99.3 & 4.3 & 100.4 & 5.7 & 98.7 & 1.9 & 99.5 & 4.1 \\
\hline Overall Mean & 97.3 & 6.3 & 100.0 & 3.1 & 99.1 & 4.4 & 100.4 & 3.9 & 99.4 & 2.8 & 99.9 & 3.4 \\
\hline
\end{tabular}

day and from the beginning to the end of the week in the 12-h/4-day week.

Analyses of variance for the total number of correct additions revealed a significant effect for week $\times$ time of shift $[F(1,4)=4.83, p<.10]$. This result reflects the improvement in performance evident across the 8-h day as opposed to the decrement in performance evident across the 12-h day (see Table 4).

Analyses of variance for the number of errors in the no-constant version of the task revealed significant interactions of group $\times$ day $\times$ time of shift $[F(5,20)=2.32$, $\mathrm{p}<.10]$ and group $\times$ week $\times$ time of shift $[\mathrm{F}(1,4)=$ $5.34, \mathrm{p}<.10]$. The group interactions are illustrated in Table 5, which presents means for each group, at each time of day, across each day in the 8-h/6-day week and the 12-h/4-day week. As shown in Table 5, errors increased in the 8-h/6-day week across each day in Group 1 and decreased in the 8-h/6-day week across each day in Group 2. In the 12-h/4-day week, however, errors increased across each day in both groups.

In summary, the results for digit addition (no constant) suggest that the 12-h/4-day week had a more detrimental effect on the performance of this task than did the 8-h/6day week. Both the number of correct additions and the number of errors were consistently poorer in both groups at the end of the longer workday. Performance across the 8-h days were not consistent across group.

The results for the digit addition task with constant were similar to the results of the task without constant. Both the speed and accuracy of performance worsened in the 12-h/4-day week and improved in the 8-h/6-day week. The results are consistent with a conclusion of greater fatigue in the 12-h/4-day week.

In addition to the performance variables described above, a number of self-report measures showed consis- tent changes in the direction of greater drowsiness or fatigue across the day by the end of the 12-h/4-day week. Changes across the 8-h days were not consistent. These measures included the Dullness or Drowsiness and Lack of Concentration subscales of the Questionnaire for Subjective Symptoms of Fatigue, the Stanford Sleepiness Scale, and the General Activation subscale of the Activation-Deactivation Adjective Checklist. The results for the Lack of Concentration Subscale shown in Table 6 illustrate the general pattern. Means and standard deviations are presented for each group across the first and last days of each workweek. Changes across the 8-h workdays and the first 12-h workday were not consistent from group to group. However, reports of inability to concentrate consistently increased across the final 12-h day in both groups. This result is supported by a significant interaction of group $\times$ day $\times$ time of shift in the 12-h/4day week $[F(1,4)=9.85, \mathrm{p}<.05]$.

\section{DISCUSSION}

Overall, the results imply that the 12 -h/4-day week was more detrimental to performance and produced more selfreports of drowsiness and fatigue than did the 8-h/6-day week. Differences between the two work schedules were apparent in the data-entry job simulation as well as in some of the brief measures of both performance and subjective state. The measures in the performance/self-report battery reflecting greater fatigue in the 12-h/4-day week included: grammatical reasoning (three-letter version), digit addition (with and without the additional constant), the Drowsiness and Lack of Concentration subscales of the Questionnaire for Subjective Symptoms of Fatigue, the Stanford Sleepiness Scale, and the General Activation subscale of the Activation-Deactivation Adjective Checklist.

Table 5

Number of Errors for Each Group in the Digit Addition Task (No Constant) (Difference from Baseline)

\begin{tabular}{|c|c|c|c|c|c|c|c|}
\hline \multirow[b]{2}{*}{ Group } & \multirow[b]{2}{*}{ Time of Shift } & \multicolumn{3}{|c|}{ 8-h Week } & \multicolumn{3}{|c|}{ 12-h Week } \\
\hline & & Day 1 & Day 6 & Mean & Day 1 & Day 4 & Mean \\
\hline \multirow{2}{*}{1} & Beginning & -1.00 & -1.33 & -1.09 & 0.33 & 1.33 & 0.83 \\
\hline & End & 0.00 & -0.33 & 0.25 & 1.83 & 1.50 & 1.67 \\
\hline 2 & Beginning & $\begin{array}{r}1.33 \\
-0.35\end{array}$ & $\begin{array}{r}1.33 \\
-250\end{array}$ & 0.83 & -1.33 & -0.33 & $\begin{array}{r}-0.83 \\
2.50\end{array}$ \\
\hline
\end{tabular}


Table 6

Group Means for the Lack of Concentration Subscale of the Questionnaire for Subjective Symptoms of Fatigue (Difference from Baseline)

\begin{tabular}{clcrrrr} 
& & \multicolumn{2}{c}{ 8-h Week } & & \multicolumn{2}{c}{ 12-h Week } \\
\cline { 7 - 8 } \cline { 6 - 7 } Group & Time of Shift & Day 1 & Day 6 & & Day 1 & Day 4 \\
\hline \multirow{2}{*}{1} & Beginning & 1.00 & 1.50 & & 2.83 & 0.50 \\
& End & 1.83 & 1.83 & & 0.17 & 1.50 \\
\multirow{2}{*}{2} & Beginning & 2.00 & 1.67 & & -0.33 & 2.67 \\
& End & 2.00 & -0.33 & & 2.67 & 4.83 \\
\hline
\end{tabular}

\section{Continuous Performance: Data Entry}

The speed of data entry increased from the beginning to the end of either workweek. It seemed easier, however, to improve efficiency in the 8-h/6-day week. This improvement is consistent with the results of Colquhoun et al. $(1968,1969)$, who reported a higher rate of improvement in calculation speed across a series of 8-h days than across a series of 12 -h days. The results of Colquhoun et al. are strengthened by the use of a repeatedmeasures design in the present investigation. This design avoided the potential confounding of hours of work with personality type that was apparent in the earlier studies (as noted by Colquhoun, personal communication, October 1982). Taken together, the two investigations favor the 8-h day as being more conducive to efficiency than the 12-h day.

Performance across the day in the present study might also be an indicator of fatigue. A statistically significant time-of-day effect was apparent in the 12-h/4-day week, in which both speed and accuracy declined from morning to late afternoon. A significant time-of-day effect was not obtained in the 8-h/6-day week. Declines in performance on the data-entry task from morning to afternoon contrast with the results of previous diurnal studies of perceptual-motor types of tasks, in which performance improved from morning to late afternoon. Other diurnal studies (see Colquhoun, 1982) have usually tested perceptual-motor abilities on an intermittent basis across the day, with less concentrated activity between testing times. There are, however, studies (Colquhoun et al., 1968, 1969; Morgan, Coates, Brown, \& Alluisi, 1973) of continuous performance that have also reported morning-to-afternoon improvements in efficiency. In contrast to the present study, these investigations required subjects to alternate between different types of tasks throughout the day. Task alternation could minimize the monotony involved and serve as a motivator to overcome fatigue. Indeed, Alluisi and Morgan (1982) reported that a simple overt demand by the experimenter to try harder can minimize (at least in the short run) the detrimental effects of continuous work combined with sleep loss. There is also reason to believe that the level of competitiveness was higher in earlier investigations. Colquhoun et al. employed military personnel as subjects, a group that is constantly encouraged to compete and strive to per- form at ever higher levels. Morgan et al. employed highly cohesive, highly trained "crews" of students, with military names such as Able and Baker, who were required to time-share tasks and adhere to militaristic regimentation. These conditions leave an impression of a competitive situation. The subjects in the present study, however, were temporary clerical service employees who did not know one another prior to the study. Although they were faithful in their duties and showed much improvement over the course of the study, there was no apparent air of intense competition.

\section{Performance/Self-Report Battery}

An impression of greater fatigue in the 12-h/4-day week was also apparent in a subset of measures in the performance/self-report battery. These included: the grammatical reasoning task (three-letter version), the digit addition task (no-constant and constant versions), the Drowsiness and Lack of Concentration subscales of the Questionnaire for Subjective Symptoms of Fatigue, the Stanford Sleepiness Scale, and the General Activtion subscale of the Activation-Deactivation Adjective Checklist.

Fatigue was apparent in a consistent slowing of response time in the grammatical reasoning task from the beginning to the end of the last 12-h workday. In the digit addition task, both speed and accuracy were less efficient at the end of the 12-h day and at the end of the 12-h/4day week. The results for the three-letter grammatical reasoning and the digit addition tasks imply a narrowing in the ability to perform logical operations (exemplified by verbal notions of order or arithmetic calculations) that place a demand on working memory (see Baddeley, 1976). More specifically, the subject was required to perform a logical operation on a letter or number string held briefly in memory and then forgotten at the initiation of the next trial. These tasks can be contrasted with the Williams Word Memory test of immediate recall, in which no differences in performance between the two work schedules were obtained. The Williams task may require different cognitive processes, since the length of the 15 word list called for a degree of organization beyond the seven-item "span of apprehension" that is assumed to limit working memory (see Baddeley, 1976). Furthermore, there was no overt logical operation applied to the stimuli in the Williams task.

Along with the tasks just described, other performance tasks showed patterns that suggested greater fatigue in the 12-h/4-day week. The lack of statistical reliability in these latter measures makes the results questionable, but the measures do warrant further experimentation. Choice reaction time was one of these tasks. The within-session standard deviation of reaction time became progressively larger across days in both the 8-h/6-day and the 12-h/4day workweeks. Larger standard deviations reflect more frequent long reaction times, which imply transient degradations in alertness while a task is being performed, or transient reductions in motor efficiency, or both. The 
results warrant replication with a greater number of subjects. The same argument applies to the lapse measure of the response alternation task. Lapses progressively increased from the beginning to the end of each week in a manner suggesting cumulative fatigue. Additionally, there were more frequent lapses in the 12-h/4-day week. Again, such a pattern of results calls for replication with a greater number of subjects.

The more consistent declines in performance across the 12-h workday were buttressed by subjective reports of increased drowsiness and inability to concentrate. Three self-report instruments closely agreed regarding these changes. Scores on the Dullness or Drowsiness and Lack of Concentration subscales of the Questionnaire for Subjective Symptoms of Fatigue and scores on the Stanford Sleepiness Scale consistently increased across the final 12-h workday. Increases in these measures across the 8-h workdays were not consistent, and decreases were often apparent. Along with these results, the General Activation subscale of the Activation-Deactivation Adjective Checklist decreased across both the 8- and 12-h workdays, but the decreases were statistically significant only in the 12-h/4-day week.

\section{Evaluation of the Battery}

In summary, at least some of the measures in the performance/self-report battery showed sensitivity to long hours of work, and others have potential for further study. The more sensitive tasks were the grammatical reasoning task, the digit addition task, and the scales of subjective alertness and fatigue, including the Questionnaire for Subjective Symptoms of Fatigue, the Stanford Sleepiness Scale, and the Activation-Deactivation Adjective Checklist. Those having potential for further study include choice reaction time and the response alernation task. Indications of greater fatigue in the data-entry task during the 12-h/4-day week provide convergent validity for the findings with the brief tasks and scales. Thus, in agreement with Volle et al. (1979), the use of a standardized measurement battery seems to be a promising approach for future studies in the field.

On the basis of the present results, a battery aimed at detecting fatigue should include at least the three-letter grammatical reasoning task, the digit addition task, and scales probing subjective alertness and ability to concentrate. Collectively, these measures suggest that reports of drowsiness and inability to concentrate after a long day reflect a deficit in the capacity to perform logical operations on information held in working memory. In considering the source of this deficit, it might be argued that it is a direct result of long hours on the data-entry task. A more plausible argument, however, would suggest that the deficit is a more general reflection of long hours of work per se. It seems unlikely that the deficit is tied to some specific component of the data-entry task itself, because that task is primarily a perceptual-motor one requir- ing a rapid, almost mechanical, translation of a stimulus into a response. It most closely resembles a five-choice reaction time task in this regard, and there is comparatively little cognitive manipulation of the stimulus before the response is made.

Future field studies utilizing the set of measures developed in the present study will evaluate long workdays in different occupations and work settings. The current status of the research, however, allows only for judgments of "more" or "less" fatigue in the comparison of different work schedules. Such a judgment could be adequate for some situations in which a switch from an 8-h day to some longer workday is being considered. In other situations, production and other economic factors, and even the preference of the workers, might necessitate tolerance of more fatigue in order to derive other benefits from the longer day. In these situations, the question of how much fatigue is allowable before the safety or health of the worker is compromised must be asked. That question can be answered in terms of the kinds of measures used in the present experiment. An adequate answer calls for the establishment of tolerance levels on these measures, presumably for different demographic groups. A score beyond the tolerance point would be judged to reflect an unsafe level of fatigue.

In conclusion, the present investigation has provided a controlled laboratory test of two overtime work schedules and has demonstrated that a 12-h/4-day week was more detrimental to performance and feelings of alertness and fatigue than an 8-h/6-day week. This was apparent both in a data-entry job simulation performed throughout the day and in brief measures of performance and subjective state. The study also laid the groundwork for a portable field-test battery for assessing long hours of work in real-life settings. The decrements in cognitive performance and the more frequent subjective complaints obtained under the 12-h/4-day schedule seemed to more generally reflect long hours of work. Thus, the battery has good potential for detecting fatigue in other situations or occupations.

\section{REFERENCES}

Alluisi, E. A., \& Morgan, B. B. (1982). Temporal factors in human performance and productivity. In E. A. Alluisi \& E. A. Fleishman (Eds.), Human performance and productivity: Vol. 3. Stress and performance effectiveness. Hillsdale, NJ: Erlbaum.

BADDELEY, A. D. (1968). A 3-min reasoning task based on grammatical transformation. Psychonomic Science, 10, 341-342.

Baddeley, A. D. (1976). The psychology of memory. New York: Basic. Bills, A. C. (1931). Blocking: A new principle in mental fatigue. American Journal of Psychology, 43, 230-245.

Campbell, L. H. (1980, April). Can new shift schedules motivate? Hydrocarbon Processing, pp. 249-256.

Chiles, W. D., Alluisi, E. A., \& Adams, O. S. (1968). Work schedules and performance during confinement. Human Factors, 9, 143-196.

Clements, P. R., Hafer, M. D., \& Vermillion, M. E. (1976). Psychometric, diurnal, and electrophysiological correlates of activation. Journal of Personality and Social Psychology, 33, 387-394. 
Colquhoun, W. P. (1982). Biological rhythms and performance. In W. B. Webb (Ed.), Biological rhythms, sleep, and performance (pp. 59-86). New York: Wiley.

Colquhoun, W. P., Blake, M. J. F., \& Edwards, R. S. (1968). Experimental studies of shiftwork II: Stabilized 8-hour shift systems. Ergonomics, 11, 527-546.

Colquhoun, W. P., Blake, M. J. F., \& Edwards, R. S. (1969). Experimental studies of shiftwork III: Stabilized 12-hour shift systems. Ergonomics, 12, 856-882.

Dember, W. N., \& WARM, J. S. (1979). Psychology of perception. New York: Holt, Rinehart \& Winston.

Ellingstad, V. S., \& Heimstra, N. W. (1970). Performance changes during the sustained operation of a complex motor task. Ergonomics, 13, 693-705.

Grandjean, E. P., Wotzka, G., Schaad, R. . \& Gilgen, A. (1971). Fatigue and stress in air traffic controllers. Ergonomics, 14, 159-165.

HEDGES, J. N. (1971, October). A look at the 4-day work week. Monthly Labor Review, pp. 33-37.

Hoddes, E., Zarcone, V., Smythe, H., Phillips, R., \& Dement, W. C. (1973). Quantification of sleepiness: A new approach. Psychophysiology, 10, 431-436.

HODGE, B. J., \& TELlier, R. D. (1975). Employee reactions to the fourday week. California Management Review, 18, 25-30.

IVANCEVICH, J. M. (1974). Effects of the shorter work week on selected satisfaction and performance measures. Journal of Applied Psychology, 59, 717-721.

IvanceVich, J. M., \& Lyon, H. L. (1977). The shortened work week: A field experiment. Journal of Applied Psychology, 62, 34-37.

Kahneman, D. (1973). Attention and effort. Englewood Cliffs, NJ: Prentice-Hall.

MONK. T. H., \& EMBreY, D. E. (1981), A field study of circadian rhythms in actual and interpolated task performance. In A. Reinberg, N. Vieux, \& P. Andlauer (Eds.), Night and shiftwork: Biological and social aspects (pp. 473-480). Oxford: Pergamon.

Morgan, B. B., Coates, G. D., Brown, B. R., \& Alluisi, E. A. (1973). Effects of continuous work and sleep loss on the recovery of sustained performance (U.S. Army Technical Memo No. 14-73). Human Engineering Laboratory, Aberdeen Proving Ground, MD.

NaIToH, P. (1981). Circadian cycles and the restorative power of naps. In L. C. Johnson, D. I. Tepas, W. P. Colquhoun, \& M. J. Colligan (Eds.), The 24-hour workday: Proceedings of a Symposium on Variations in Work-Sleep Schedules (pp. 693-720). Cincinnati: U.S. Department of Health and Human Services.

NORD, W. R., \& COSTIGAN, R. (1973). Worker adjustment to the fourday week: A longitudinal study. Journal of Applied Psychology, 58, 60-66.

THAYER, R. E. (1967). Measurement of activation through self-report. Psychological Reports, 20, 663-678.

ThORNDIKE, E. L., \& LORGE, I. (1944). The teacher's word book of 30,000 words. New York: Teachers College Press, Columbia University.

Volle, M., Brisson, G. R., Perusse, N., Tanaka, M., \& Doyon, Y. (1979). Compressed work-week: Psychophysiological and physiological repercussions. Ergonomics, 22, 1001-1010.

Williams, H. L., Geiseking, C., \& Lubin, A. (1966). Some effects of sleep loss on memory. Perceptual and Motor Skills, 23, 1287-1293.

WiLliams, H. L., \& LUBin, A. (1966). Speeded addition and sleep loss. Journal of Experimental Psychology, 73, 313-317.

Yoshitake. H. (1971). Relationship between the symptoms and the feeling of fatigue. Ergonomics, 14, 175-186.

Yoshit AKE, H. (1978). Three characteristic patterns of subjective fatigue symptoms. Ergonomics, 21, 231-233. 